\title{
Pterostilbene Improves Cognitive Performance in Aged Rats: An in Vivo Study
}

\author{
Martina La Spina ${ }^{a, b} \quad$ Gabriele Sansevero ${ }^{c, d} \quad$ Lucia Biasutto $^{a, b}$ Mario Zoratti ${ }^{a, b}$ \\ Roberta Peruzzo ${ }^{e}$ Nicoletta Berardic,d Alessandro Sale ${ }^{c}$ Michele Azzolini ${ }^{a, b}$ \\ a University of Padova, Dept. of Biomedical Sciences, Padova, Italy, ${ }^{\mathrm{b} C N R}$ Neuroscience Institute, Padova, \\ Italy, 'CNR Neuroscience Institute, Pisa, Italy, dUniversity of Florence, Dept. Neuroscience, Psychology, \\ Drug Research and Child Health (Neurofarba), Florence, Italy, eUniversity of Padova, Dept. of Biology, \\ Padova, Italy
}

\section{Key Words}

Pterostilbene $\cdot$ Aging $\cdot$ Cognitive decline $\cdot$ T-maze $\cdot$ Object recognition $•$ Object in context $•$ Synaptic plasticity

\begin{abstract}
Background/Aims: Pterostilbene ( $\mathrm{Pt}$; trans-3,5-dimethoxy-4'-hydroxystilbene) is a natural phenol found in blueberries and grapevines. It shows remarkable biomedical activities similar to those of resveratrol. Its high bioavailability is a major advantage for possible biomedical applications. The goal of the study was to evaluate the effects of chronic pterostilbene administration on cognitive performance in aged rats with mild cognitive impairment. Methods: 18-month-old animals were subjected to behavioral tests to establish the "baseline", then divided into treatment and control groups. The former were chronically fed Pt ( $22.5 \mathrm{mg} / \mathrm{kg}$-day) for 20 consecutive days. At the end of this period all animals were tested again and sacrificed. The dentate gyrus, the hippocampus and the prefrontal and perirhinal cortices were then collected, and RT-qPCR and/or Western blot analyses were performed on a few transcripts/proteins involved in synaptic remodeling. Mitochondrial content was also assessed. Results: Pt administration improved performance in behavioral tests and positively affected memory consolidation. We found increased levels of REST, PSD-95 and mitochondrial porin1 in the dentate gyrus and a positive correlation between T-maze test score and levels of CAMP responsive element binding protein (CREB) phosphorylation. Conclusion: These results underscore the therapeutic potential of Pt supplementation for age-related cognitive decline.
\end{abstract}

M. La Spina and G. Sansevero contributed equally to this work. 


\section{Introduction}

Intake of fruits and other plant-derived foods, and in particular of berries and nuts, antagonizes the progressive cognitive decline associated with aging and neurodegenerative disorders [1-3] and with the metabolic syndrome [1,2]. These effects are largely attributed to the polyphenolic components of these foods, including flavonoids [3] and stilbenoids [4]. They are thought to act by multiple mechanisms, including antagonizing neuroinflammation $[3,5]$, improving blood circulation [6] and increasing neurogenesis and brain plasticity [7].

A major issue in the field is bioavailability and brain accessibility in vivo $[3,8]$. Dietary polyphenols, being ready-made substrates for Phase II enzymes, are rapidly transformed into metabolites in enterocytes, hepatocytes, and in the gut due to the action of bacteria [9]. These metabolites are rapidly excreted, and metabolic modification is generally considered to lower efficacy. The most studied stilbenoid, resveratrol, is rapidly and heavily modified by sulpho- and glucurono-transferases [10]. Its dimethylated analog, pterostilbene (trans-3, 5-dimethoxy-4'-hydroxystilbene), present in berries, is less susceptible to modifications, more bioavailable, and reaches relatively high (several nanomoles/gram) levels in the brain tissue after oral administration of moderate pharmacological doses [11]. Moreover, it is considered completely safe [12]. This favorable bioavailability profile can be further improved using prodrugs conjugating the molecule with natural amino acids [13]. Indeed, administration of pterostilbene has been reported to support memory formation or maintenance [14], and to be more effective than resveratrol in this respect [15]. In the report by Joseph et al. [14] the improvement could be correlated with the levels of pterostilbene found in the hippocampus of the animals. The present paper also deals with potentiation of cognitive functions by pterostilbene. We briefly discuss here a few aspects of direct relevance.

Age-related losses are substantial in tasks of declarative memory and working memory. Declarative memory formation and consolidation is largely dependent on the hippocampus [16], a brain region strongly affected by aging [17]; working memory is dependent on the prefrontal cortex, another brain region altered by aging [18]. It proceeds via a short-term phase, in which newly encoded information can be retained for, at most, several hours, followed by a consolidation process that generates a stable long-term memory trace [19]. This process comprises de novo gene expression and protein synthesis, and therefore relies on the activities of transcription factors, among which CREB, activated by phosphorylation at Ser133, has been demonstrated to play a central role, promoting adult hippocampal neurogenesis in the dentate gyrus (DG) [20]. Another transcription factor of relevance for cognitive functions is REST (RE1-silencing transcription factor), highly expressed in the hippocampus, which shows a complex behavior during development and aging. It acts as a key regulator (repressor) of neuronal gene expression, but it also has distinct protective roles: its levels in old age correlate with cognitive preservation and longevity, and it is upregulated by physical exercise $[21,22]$.

Post-synaptic density protein 95 (PSD-95) has a fundamental role in the organization of the signal transduction apparatus at synapses, and it is therefore of special relevance in plasticity, i.e., learning and memory formation [23]. It shows complex variation patterns in aging and in neuropathologies, but, for example, it has been found to increase in rodents exposed to an enriched environment [24], a promising strategy to mitigate cognitive decline and Alzheimer's disease dysfunctions [25]. Mitochondria are deeply involved in neuronal plasticity [26] and neurogenesis [27], providing ATP for processes involved in neurite outgrowth. Conversely, a reduction of mitochondrial turn-over is associated with the cognitive decline of aging and neurodegenerative disorders [28], and may be ascribed to alterations in degradative pathways. Memory consolidation may thus be expected to be accompanied by changes of the mitochondrial network.

We report here the results of a study aimed at evaluating whether a chronic administration of pterostilbene could improve cognitive performance in aged rats and at assessing pterostilbene-induced molecular modifications in the hippocampus and in the prefrontal and perirhinal cortices. 


\section{Cellular Physiology Cell Physiol Biochem 2019;52:232-239 \begin{tabular}{ll|l} 
and Biochemistry & $\begin{array}{l}\text { DOI: 10.33594/000000017 } \\
\text { Published online: 28 February } 2019\end{array}$ & $\begin{array}{l}\text { C } 2019 \text { The Author(s). Published by } \\
\text { Cell Physiol Biochem Press GmbH\&Co. KG }\end{array}$ \\
\hline
\end{tabular}}

La Spina et al.: Pterostilbene Improves Cognitive Performance in Aged Rats

\section{Materials and Methods}

Only a brief summary is given here. Full details can be found in the Supplementary Material file (all supplementary material available online at www.cellphysiolbiochem.com).

Pterostilbene administration to 18-month-old rats was by inclusion of the compound in palatable jellies [29] which were provided twice a day (12-hour intervals) and immediately consumed, for 20 consecutive days. Dosage was $22.5 \mathrm{mg} / \mathrm{kg}$ b.w. each jelly cake. Controls received "unloaded" jelly.

The cognitive performance of the animals was evaluated before and after the pterostilbene administration period using a working memory test (T-maze; [30]) and two declarative memory tests (visual object recognition - vORT- and object-in-context - OCT; [31]). After the final round of testing, the animals were sacrificed, and the dentate gyrus (DG), the rest of the hippocampus and prefrontal and perirhinal cortices were dissected, immediately frozen and stored at $-80^{\circ} \mathrm{C}$ for subsequent analysis. The mean of the arm alternations was used as evaluation criterion for the T-maze experiments. The preference to enter one arm rather than the other one was compared across groups (treated and untreated). For vORT and OCT, the time spent exploring the objects was measured. A discrimination index (DI) was calculated as the difference between the time spent exploring new and old objects divided by the total time spent exploring the objects. In all cases the indexes calculated were used for comparison across different groups (treated and untreated) with the two-way repeated measures ANOVA and the Holm-Sidak method for post hoc comparison.

For RT-qPCR and Western Blotting total RNA and proteins extraction was performed with TRIzol (Invitrogen). Reverse transcription was performed using the SuperScript VILO cDNA Synthesis kit. The specific primers used to measure the transcription of genes of interest are listed in Table S1. IQ Syber Green Supermix (Biorad) was used for detection. Relative quantification of target genes was performed as in [32]. GAPDH was used as reference gene. Primary antibodies used for Western blotting are reported in Table S2. Odissey Imaging equipment (LICOR) was used as detection system.

In vitro experiments were performed with HEK293 cells incubated or not with pterostilbene in DMEM and then lysed.

\section{Ethics Statement}

All in vivo procedures met the requirements of Italian law (Laws 116/92 and 26/14) and of the European Community Council (Directives 86/609/EEC and 210/63/UE) and were approved by the Italian Ministry of Health (DM n. 282/2013-B).

\section{Results}

\section{Behavioral tests}

We examined the effect of a chronic administration of pterostilbene on the behavioral performances of 18-month-old rats. Since the hippocampus, and in particular the dentate gyrus, is known to be particularly vulnerable to aging [17], we chose to perform those tests that could mirror activities in these areas of the brain, such as T-maze and the object in context recognition test (OCT). Moreover, we used the visual object recognition test (vORT) to also include other cognitive processes not directly linked to the hippocampus, but mainly to two distinct cortical areas (i.e., perirhinal and prefrontal cortices). The T-maze test assesses working memory and alternation performance, and it has been demonstrated to be susceptible to hippocampal impairment [30]. We demonstrated that Pt-treated rats did not suffer from detrimental effects in the ability to alternate between the two arms and reached a statistically significant better performance than control animals $(p=0.596$ and $p=0.013$, respectively) (Fig. 1A). The OCT is specific for the assessment of memory stabilization, requiring an intact hippocampal function. OCT evaluates the ability of the animals to associate objects with contexts and to discriminate between the object when presented in a changed context and the same object presented in the same context. As shown in Fig. 1B, Pt-treated rats exhibited a significantly higher score compared to baseline levels $(\mathrm{p}=0.002)$. Conversely, the performance of untreated rats did not change compared to that observed 
during the baseline evaluation ( $\mathrm{p}=0.553$ ). The vORT is aimed at assessing memory retention abilities regarding information deriving from the environment and orientation (declarative memory). vORT is based on the spontaneous tendency of rodents to spend more time exploring a novel object than a familiar one, and it also involves hippocampal function. It was performed twice, 1 and 24 hours after the training sessions, with similar results. At both times, animals chronically treated with $\mathrm{Pt}$ explored the new object more than rats belonging to the control group $(\mathrm{p}<0.001$ and $\mathrm{p}$ $=0.002$, respectively) (Fig. $1 \mathrm{C}$, D).

\section{Molecular readouts of} pterostilbene treatment

As the DG is wrapped up by the surrounding anatomical structures of the hippocampus, its dissection is notoriously difficult. To limit problems related to tissue contamination, we verified the accuracy of the procedure as detailed in Supplementary Fig. S1. Hereafter we use the term "hippocampus" to refer to the excised cerebral tissue

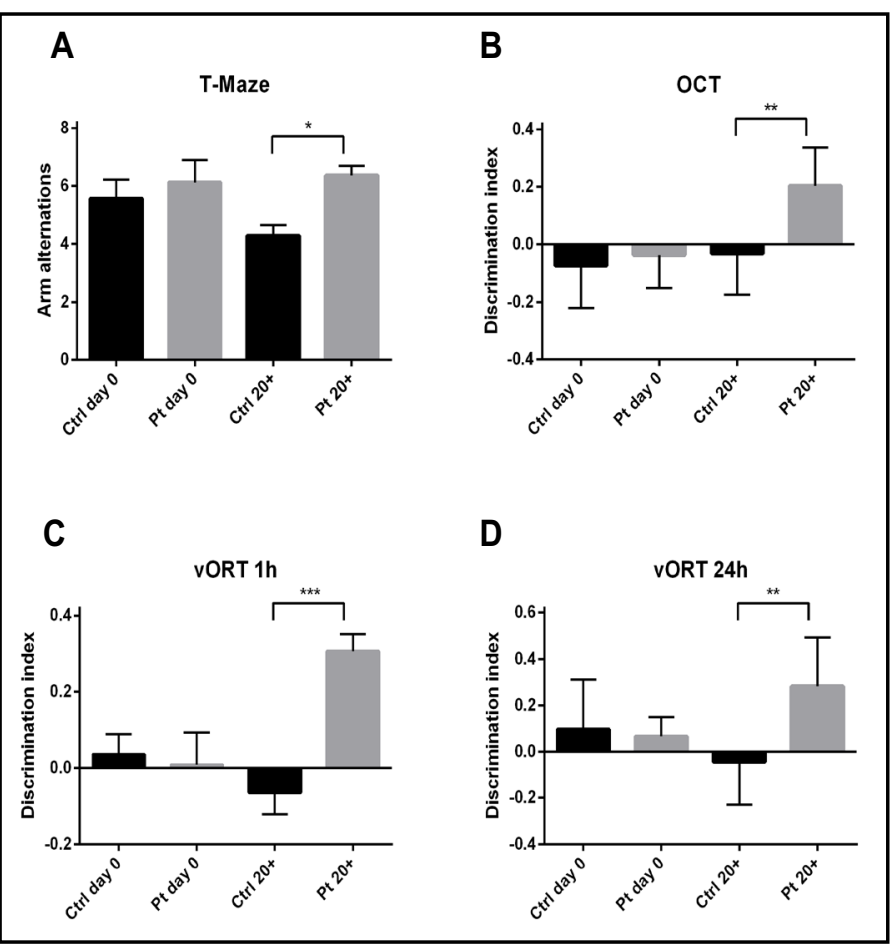

Fig. 1. Behavioral assessment of pterostilbene administration effects on cognitive abilities. (A) T-Maze: while there was no alternation difference between the two groups before the treatment ( $p=0.619$ ), Pt-treated animals performed better than controls after pterostilbene administration ( $p=0.013)$. (B) OCT: the Pttreated group was able to discriminate between the two objects significantly better than the control group ( $p=0.003)$. (C, D) vORT: Pt-treated rats improved their performance compared to control rats, and were able to discriminate between the two objects at both 1 and 24 hours $(\mathrm{p}<0.001$ and $\mathrm{p}=0.002$, respectively). Histograms represent average values $\pm \mathrm{SEM} .{ }^{*}, \mathrm{p}<0.05 ;{ }^{* *}, \mathrm{p}<0.01 ;{ }^{* *}, \mathrm{p}<0.001$. Two-way RM ANOVA, Holm-Sidak method. $\mathrm{N}=7$ for the control group and $\mathrm{N}=8$ for the treated group. containing the hippocampus minus the DG. Given their involvement, both the prefrontal and the perirhinal cortices were also collected and analysed. We examined a set of transcripts and proteins that may be involved in synaptic plasticity processes (see Supplementary Table S1 and S2 for a complete list). While the average increase of phospho-CREB (pCREB) in the DG of Pt-treated animals was only marginally significant $(p=0.0886)($ Fig. $2 A)$, a positive correlation $(p=0.0105)$ was obtained between these levels and the logarithm of the score obtained by each treated animal in the T-maze test (Fig. 2D). No such correlation was found for the control group (Fig. 2C), in agreement with the idea that cognitive decline during aging is multi-factorial, not ascribable to a single factor.

To further assess the impact of Pt on CREB, we also verified whether it could activate CREB in vitro. Western blot analysis performed in HEK293 cells confirmed that Pt induced an increase in the phosphorylation levels of CREB, with a maximal effect reached after about 20 - 45 minutes (Fig. 3).

Additionally, we also observed higher transcription levels of neuronal regulator REST in treated animals (Fig. 4A) compared to controls, further supporting the idea that Pt may induce changes in gene expression leading to rearrangement in synaptic structures. The results we obtained both in vitro and in vivo led us to speculate that Pt may actually promote 
the remodelling of synaptic structures and the establishment of new neuronal connections.

On this basis, we further investigated possible changes induced by $\mathrm{Pt}$ at the synaptic level. We focused on postsynaptic density protein 95 (PSD-95) as it represents a major marker of synaptic maturation and it plays a key role in the stabilization of modifications of synaptic structures underlying functional activity-related changes, such as memory formation and consolidation. Importantly, the expression of this protein was significantly higher in the animals that had received $\mathrm{Pt}$ than in the control group (Fig. 4B).

Neuronal rewiring requires energy. Thus, we also focused our analysis on cellular mitochondrial content, as mitochondria are the main energy suppliers in cells. Western blot analysis showed a higher amount of porin 1, a protein of the mitochondrial outer membrane used as a classical mitochondrial marker, in the DG of treated rats (Fig. 4C).

All the investigations we just described were also carried out on prefrontal and perirhinal cortices samples. However, Pt treatment seemed to have no effect in these areas of the brain (not shown).

\section{Discussion}

The increase in the levels of PSD95 (Fig. 4B) suggests that Pt may influence synaptic strength and morphology inducing Long-Term Potentiation (LTP), which, along with specific variations in cell excitability, underlies memory formation and consolidation [33]. Multiple factors and proteins contribute to the process, with a key role by $\mathrm{Ca}^{2+} /$ calmodulindependent protein kinases. Interestingly, low- $\mu \mathrm{M}$ concentrations of pterostilbene have been reported to mobilize $\mathrm{Ca}^{2+}$ in cultured cells [34].
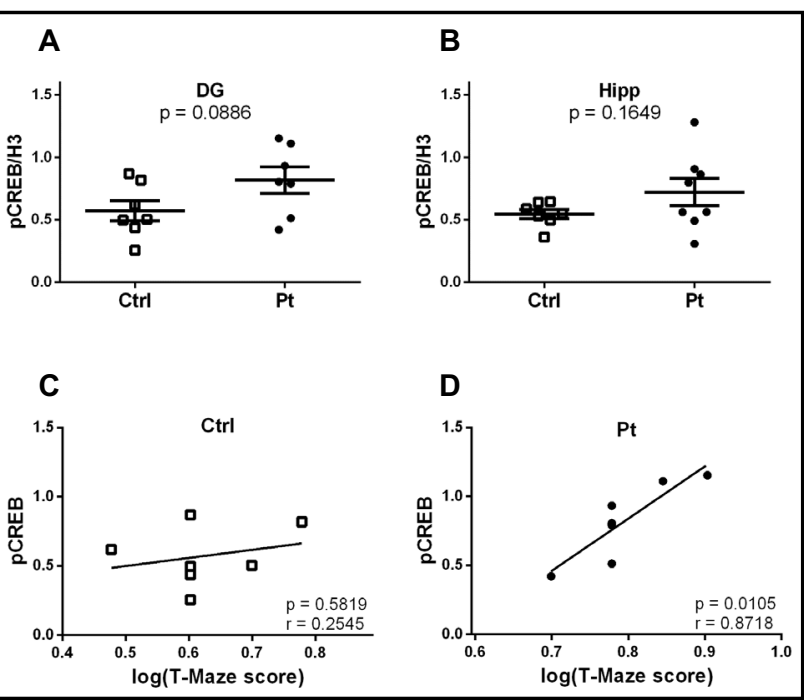

Fig. 2. (A, B): Western blot analysis of pCREB. Pt treatment may stimulate the activation of the transcription factor specifically in the dentate gyrus of 18-month-old rats. Statistical analysis performed with two-tailed unpaired t-test. $\mathrm{N}=7$. Error bars represent SEM. (C, D): Correlations between the scores obtained in the T-maze behavioral test and pCREB levels in the dentate gyrus of control (C) and Pt-treated (D) ats. $\mathrm{N}=7$.

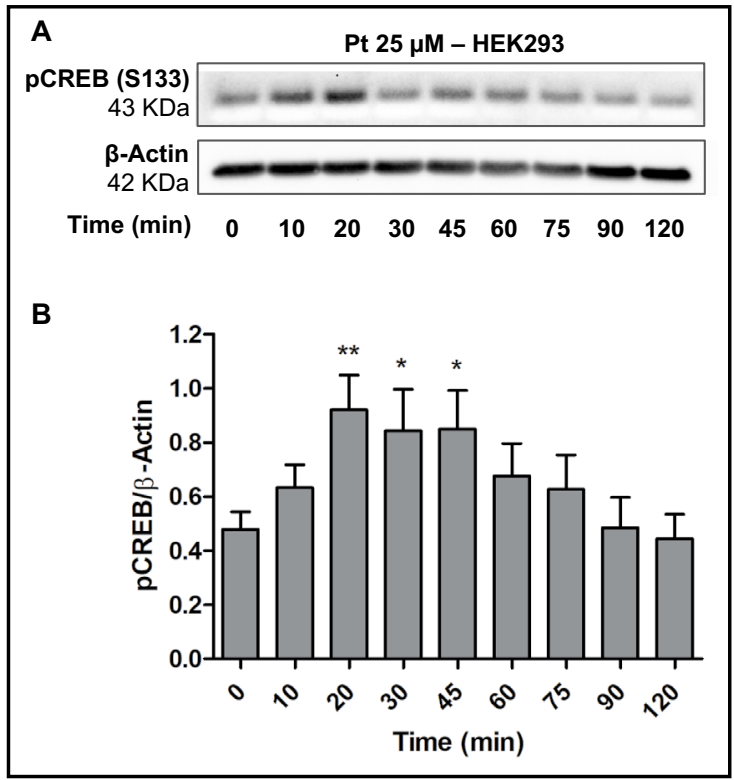

Fig. 3. Western Blot analysis of pCREB. (A): a representative Western Blot. (B): histogram of the average densitometry data. Statistics: Repeated measures one way ANOVA test with Dunnett's correction. ${ }^{*}, * *$ : $\mathrm{p} \leq 0.05, \leq 0.01$ with respect to control $(t=0)$, respectively. $N=7$. Error bars represent + SEM. 
The effects of pterostilbene treatment may extend to the promotion of mitochondrial biogenesis (Fig. 4C). While not investigated, to our knowledge, in the case of pterostilbene, PGC- $1 \alpha$, the master regulator of this process, has been reported to be upregulated by resveratrol also in vivo, likely downstream of SIRT1 action and/or NO/CO signaling [35]. Dendritic mitochondria have been proposed [36] to participate to hippocampal synaptic LTP - more precisely to late-phase structural LTP via the generation of "mitoflashes", i.e. bursts of mitochondrial ROS production accompanied by mitochondrial depolarization associated with opening of the Mitochondrial Permeability Transition Pore (MPTP) [37]. It may be relevant in this context that pterostilbene, despite being generally considered an antioxidant, can induce ROS production and mitochondrial depolarization [38].

While several observations and reports thus suggest that pterostilbene may act directly on nervous cells, the possibility remains that its behavioral effects may be, wholly or in part, indirect, mediated by an improvement of the oxygen supply to the hippocampus. This compound, like resveratrol, can in fact stimulate NO production by eNOS, thus inducing vasodilation [39]. While this possibility (and the possibility of multiple modes of action) remains to be explored, it is relevant that we observed CREB phosphorylation, one of the phenomena involved in LTP, in cultured (i.e., well- or even over-oxygenated) cells.

This is only the second study (the other being [14]) examining the effect of pterostilbene on cognitive performance carried out with naturally

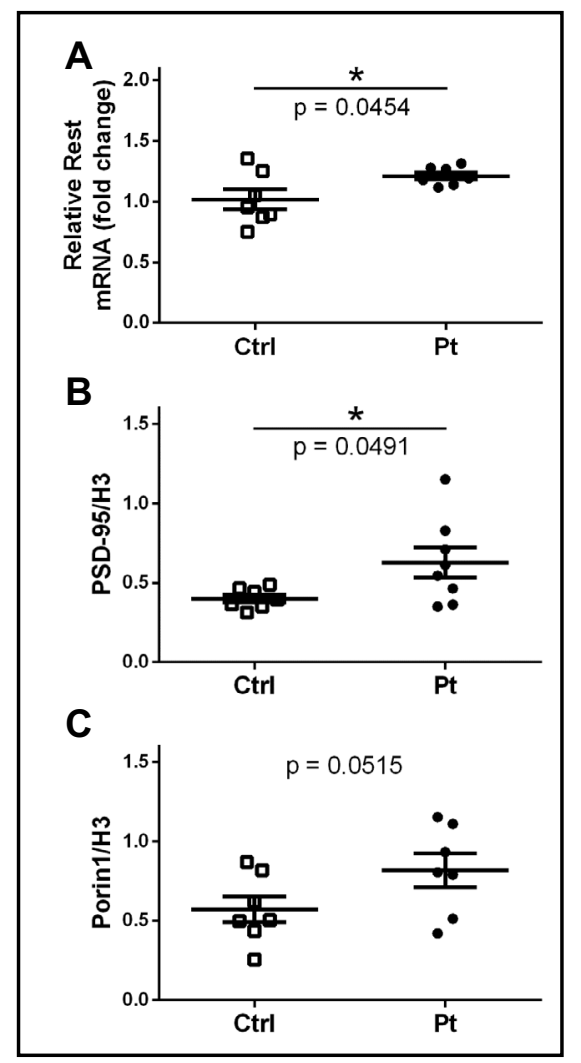

Fig. 4. (A): mean values of REST mRNA in the dentate gyrus of 18-month-old rats. ( $\mathrm{B}$ and $\mathrm{C}$ ): protein expression of PSD-95 (B) and Porin1 (C). *: p<0.05. Statistical analysis performed with the two-tailed unpaired t-test. $N \geq 7$. Error bars represent SEM. aged rodents, as distinct from progeroid or other genetic models and from models of drug, hypoxia- or trauma-induced CNS dysfunction. The findings, which broadly agree with and reinforce those of Joseph et al. [14] strongly suggest that moderate and chronically administered doses of pterostilbene may antagonize age-related cognitive decline. Although the molecular mechanisms underlying these effects remain to be completely defined, we have obtained evidence pointing to the activation of key transcription factors (CREB, REST) and to the upregulation of components of cellular structures (synapses, mitochondria) involved in memory formation and consolidation, pointing to stimulation of brain plasticity as the final mechanism underlying the beneficial effects of Pt. Pterostilbene was selected for this study because of its remarkable bioavailability and of the high levels it can reach in the brain. The results warrant further explorations aimed at the optimisation of a potentially very helpful, safe and cheap nutritional "brain-aid".

\section{Acknowledgements}

We thank Laura Baroncelli for training in the brain dissection work, Sandra Furlan for help in setting up the protocols for RT-qPCR analyses, Paolo Bernardi for support and discussions. This work was financed by the CNR Project of Special Interest on Aging, by the CNR InterOmics project, by the Italian Ministry of Education, Universities and Research 
La Spina et al.: Pterostilbene Improves Cognitive Performance in Aged Rats

(MIUR) (PRONAT project) and by the Italian Association for Cancer Research (AIRC) (IG grant 15544). M.A. gratefully acknowledges support by a fellowship from Fondazione Umberto Veronesi. M.L.S. was partly supported by a fellowship from Telethon grant MitCare-2 (GGP14187).

\section{Disclosure Statement}

The authors have no conflicts of interest to declare.

\section{References}

1 Miller MG, Thangthaeng N, Poulose SM, Shukitt-Hale B: Role of fruits, nuts, and vegetables in maintaining cognitive health. Exp Gerontol 2017;94:24-28.

2 Carey AN, Galli RL: Mitigating the effects of high fat diet on the brain and behavior with berry supplementation. Food Funct 2017;8:3869-3878.

3 Jaeger BN, Parylak SL, Gage FH: Mechanisms of dietary flavonoid action in neuronal function and neuroinflammation. Mol Aspects Med 2018;61:50-62.

4 Poulose SM, Thangthaeng N, Miller MG, Shukitt-Hale B: Effects of pterostilbene and resveratrol on brain and behavior. Neurochem Int 2015;89:227-233.

5 Sawikr Y, Yarla NS, Peluso I, Kamal MA, Aliev G, Bishayee A: Neuroinflammation in Alzheimer's Disease: The Preventive and Therapeutic Potential of Polyphenolic Nutraceuticals. Adv Protein Chem Struct Biol 2017;108:33-57.

- 6 Ghosh D, Scheepens A: Vascular action of polyphenols. Mol Nutr Food Res 2009;53:322-331.

- 7 Poulose SM, Miller MG, Scott T, Shukitt-Hale B: Nutritional Factors Affecting Adult Neurogenesis and Cognitive Function. Adv Nutr 2017;8:804-811.

8 Pandareesh MD, Mythri RB, Srinivas Bharath MM: Bioavailability of dietary polyphenols: Factors contributing to their clinical application in CNS diseases. Neurochem Int 2015;89:198-208.

9 Scalbert A, Morand C, Manach C, Remesy C: Absorption and metabolism of polyphenols in the gut and impact on health. Biomed Pharmacother 2002;56:276-282.

10 Wenzel E, Somoza V: Metabolism and bioavailability of trans-resveratrol. Mol Nutr Food Res 2005;49:472481.

11 Azzolini M, La Spina M, Mattarei A, Paradisi C, Zoratti M, Biasutto L: Pharmacokinetics and tissue distribution of pterostilbene in the rat. Mol Nutr Food Res 2014;58:2122-2132.

- 12 Ruiz MJ, Fernandez M, Pico Y, Manes J, Asensi M, Carda C, Asensio G, Estrela JM: Dietary administration of high doses of pterostilbene and quercetin to mice is not toxic. J Agric Food Chem 2009;57:3180-3186.

13 Azzolini M, Mattarei A, La Spina M, Fanin M, Chiodarelli G, Romio M, Zoratti M, Paradisi C, Biasutto L: New natural amino acid-bearing prodrugs boost pterostilbene's oral pharmacokinetic and distribution profile. Eur J Pharm Biopharm 2017;115:149-158.

14 Joseph JA, Fisher DR, Cheng V, Rimando AM, Shukitt-Hale B: Cellular and behavioral effects of stilbene resveratrol analogues: implications for reducing the deleterious effects of aging. J Agric Food Chem 2008;56:10544-10551.

15 Chang J, Rimando A, Pallas M, Camins A, Porquet D, Reeves J, Shukitt-Hale B, Smith MA, Joseph JA, Casadesus G: Low-dose pterostilbene, but not resveratrol, is a potent neuromodulator in aging and Alzheimer's disease. Neurobiol Aging 2012;33:2062-2071.

16 Voss JL, Bridge DJ, Cohen NJ, Walker JA: A Closer Look at the Hippocampus and Memory. Trends Cogn Sci 2017;21:577-588.

17 Bettio LEB, Rajendran L, Gil-Mohapel J: The effects of aging in the hippocampus and cognitive decline. Neurosci Biobehav Rev 2017;79:66-86.

18 Park DC, Reuter-Lorenz P: The adaptive brain: aging and neurocognitive scaffolding. Annu Rev Psychol 2009;60:173-196.

19 Miranda M, Bekinschtein P: Plasticity Mechanisms of Memory Consolidation and Reconsolidation in the Perirhinal Cortex. Neuroscience 2018;370:46-61. 


\section{Cellular Physiology Cell Physiol Biochem 2019;52:232-239

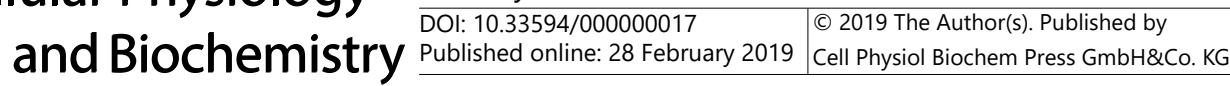

La Spina et al.: Pterostilbene Improves Cognitive Performance in Aged Rats

- 20 Kandel ER: The molecular biology of memory: cAMP, PKA, CRE, CREB-1, CREB-2, and CPEB. Mol Brain 2012;5:14.

21 Thiel G, Ekici M, Rossler OG: RE-1 silencing transcription factor (REST): a regulator of neuronal development and neuronal/endocrine function. Cell Tissue Res 2015;359:99-109.

- 22 Zhao Y, Zhu M, Yu Y, Qiu L, Zhang Y, He L, Zhang J: Brain REST/NRSF Is Not Only a Silent Repressor but Also an Active Protector. Mol Neurobiol 2017;54:541-550.

- 23 Savioz A, Leuba G, Vallet PG: A framework to understand the variations of PSD-95 expression in brain aging and in Alzheimer's disease. Ageing Res Rev 2014;18:86-94.

24 Nithianantharajah J, Levis H, Murphy M: Environmental enrichment results in cortical and subcortical changes in levels of synaptophysin and PSD-95 proteins. Neurobiol Learn Mem 2004;81:200-210.

25 Sansevero G, Sale A: Environment as therapy: neuroscience for intellectual disability and dementia. Oncotarget 2017;8:5682-5683.

26 Todorova V, Blokland A: Mitochondria and Synaptic Plasticity in the Mature and Aging Nervous System. Curr Neuropharmacol 2017;15:166-173.

27 Beckervordersandforth R, Ebert B, Schaffner I, Moss J, Fiebig C, Shin J, Moore DL, Ghosh L, Trinchero MF, Stockburger C, Friedland K, Steib K, von Wittgenstein J, Keiner S, Redecker C, Hölter SM, Xiang W, Wurst W, Jagasia R, Schinder AF: Role of Mitochondrial Metabolism in the Control of Early Lineage Progression and Aging Phenotypes in Adult Hippocampal Neurogenesis. Neuron 2017;93:560-573.e6.

- 28 Grimm A, Eckert A: Brain aging and neurodegeneration: from a mitochondrial point of view. J Neurochem 2017;143:418-431.

- 29 Zhang L: Voluntary oral administration of drugs in mice. Protoc Exch 2011; DOI:10.1038/protex.2011.236.

- 30 Deacon RM, Rawlins JN: T-maze alternation in the rodent. Nat Protoc 2006;1:7-12.

- 31 De Rosa R, Garcia AA, Braschi C, Capsoni S, Maffei L, Berardi N, Cattaneo A: Intranasal administration of nerve growth factor (NGF) rescues recognition memory deficits in AD11 anti-NGF transgenic mice. Proc Natl Acad Sci USA 2005;102:3811-3816.

32 Pfaffl MW: A new mathematical model for relative quantification in real-time RT-PCR. Nucleic Acids Res 2001;29:e45.

33 Lisman J, Cooper K, Sehgal M, Silva AJ: Memory formation depends on both synapse-specific modifications of synaptic strength and cell-specific increases in excitability. Nat Neurosci 2018;21:309-314.

34 Zhang L, Cui L, Zhou G, Jing H, Guo Y, Sun W: Pterostilbene, a natural small-molecular compound, promotes cytoprotective macroautophagy in vascular endothelial cells. J Nutr Biochem 2013;24:903-911.

35 Muhammad MH, Allam MM: Resveratrol and/or exercise training counteract aging-associated decline of physical endurance in aged mice; targeting mitochondrial biogenesis and function. J Physiol Sci 2018;68:681-688.

36 Fu ZX, Tan X, Fang H, Lau PM, Wang X, Cheng H, Bi GQ: Dendritic mitoflash as a putative signal for stabilizing long-term synaptic plasticity. Nat Commun 2017;8:31.

37 Wang W, Fang H, Groom L, Cheng A, Zhang W, Liu J, Wang X, Li K, Han P, Zheng M, Yin J, Wang W, Mattson MP, Kao JP, Lakatta EG, Sheu SS, Ouyang K, Chen J, Dirksen RT, Cheng H: Superoxide flashes in single mitochondria. Cell 2008;134:279-290.

38 Alosi JA, McDonald DE, Schneider JS, Privette AR, McFadden DW: Pterostilbene inhibits breast cancer in vitro through mitochondrial depolarization and induction of caspase-dependent apoptosis. J Surg Res 2010;161:195-201.

39 Park SH, Jeong SO, Chung HT, Pae HO: Pterostilbene, an Active Constituent of Blueberries, Stimulates Nitric Oxide Production via Activation of Endothelial Nitric Oxide Synthase in Human Umbilical Vein Endothelial Cells. Plant Foods Hum Nutr 2015;70:263-268. 